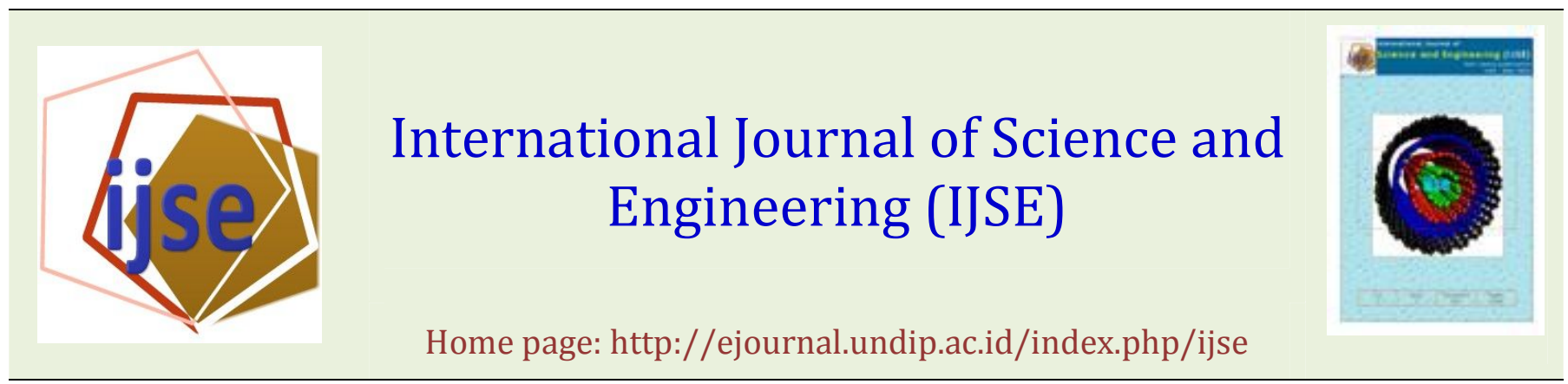

\title{
Weighted Local Active Pixel Pattern (WLAPP) for Face Recognition in Parallel Computation Environment
}

\author{
Mallikarjuna Rao $G^{1, *}$ and Jella Rajesham², \# \\ * Department of CSE, Gokaraju Rangaraju Institute of Engineering \&Technology, Autonomous under JNTUH, \\ Hyderabad, India. \\ 1. gmr333@yahoo.com \\ \# Department of CSE, Gokaraju Rangaraju Institute of Engineering \&Technology, Autonomous under JNTUH, \\ Hyderabad, India. \\ 2. rajeshamj@gmail.com
}

\begin{abstract}
The availability of multi-core technology resulted totally new computational era. Researchers are keen to explore available potential in state of art-machines for breaking the bearer imposed by serial computation. Face Recognition is one of the challenging applications on so ever computational environment. The main difficulty of traditional Face Recognition algorithms is lack of the scalability. In this paper Weighted Local Active Pixel Pattern (WLAPP), a new scalable Face Recognition Algorithm suitable for parallel environment is proposed. Local Active Pixel Pattern (LAPP) is found to be simple and computational inexpensive compare to Local Binary Patterns (LBP). WLAPP is developed based on concept of LAPP. The experimentation is performed on FG-Net Aging Database with deliberately introduced $20 \%$ distortion and the results are encouraging.
\end{abstract}

Keywords - Active pixels, Face Recognition, Local Binary Pattern (LBP), Local Active Pixel Pattern (LAPP), Pattern computing, parallel workers, template, weight computation.

$\begin{array}{lll}\text { Submission : July 23, } 2013 & \text { Corrected : October 5, 2013 Accepted: October 11, 2013 }\end{array}$

Doi: $10.12777 /$ ijse.5.2.41-46

How to cite this article: Rao G., M. and Rajesham, J. (2013). Weighted Local Active Pixel Pattern (WLAPP) for Face Recognition in Parallel Computation Environment. . International Journal of Science and Engineering, 5(2),18-24. Doi: 10.12777/ijse.5.2.41-46]

\section{INTRODUCTION}

Traditional way of programmability and serial execution has almost reached its limit. The goal new trend is not only programmability but also parallelism to meet going demands of high performance computing applications in audio, video, gaming, control systems, network applications and network security. The open source APIs (Open CL, Aparapi, and C++ AMP) for software developers will enable to take the available hardware computational power. Open MP and MPI are used to perform the parallel computation on SIMD machines.

Vision based applications need more computational power, time and space. However, the traditional approaches algorithms are inherently based on serial computation (SISD) they are not able to extract available computational power of state of art machines/devices. Net result is not only wastage of resources but also poor ROI(Return of Investment). As the technological advancements in VHLSI is resulted availability of high computational gadgets to the reach of large volume of users. For example quad-core mobiles like Cherry Mobile Sky Fire 2.0, Starmobile Knight and Starmobile Diamond V7 are available for few thousand bucks. The developers are forced to dump tons of applications into these devices. This situation forced the researchers to revise the traditional serial based techniques so that they can be scalable and support parallel implementation. In this regard there is not much progress especially Face Recognition domain. The dependency among the computational parameters of PCA and ICA etc., makes them hard to be converted and to be deployed on parallel machines. The scalability of LAPP $[5,6,7]$ Face Recognition 
Algorithm is encouraged us to extend WLAPP for the parallel environment.

Parallel models for Face Recognition [2] are proposed by Heng Fui Liau, Kah Phooi Seng, Li-Minn Ang and Siew Wen Chin, University of Nottingham Malaysia Campus Malaysia and different variants of Linear Discriminant Analysis(LDA) [2] are discussed. The authors [2] are applied weight concept to the features derived from spatial and frequency domains while developing parallel model using LDA.

The traditional algorithms such as LDA, ICA, FLD are exposed to SSS problem [2]. SSS problem is the result of the singularly of the computed scattered matrix due to the limited samples/feature values.

Parallel associative memory based efficient face recognition system [3] is also proposed by authors [3] to achieve high recognition rate. The system has been implemented using auto-associative memory blocks in parallel. The goal is to scale the associative memories to high resolution images so that discriminative features are retained and benefits of associative memory are applied to face recognition. The authors [3] employed parallel feature computation using associate-memory; however the extension to the bulk database is not effective enough in terms of space and time computations.

Paper Organisation: Face Recognition approaches to parallel environment are dealt in section 2. In the section 3 LAPP and Parallel LAPP is proposed. Section 4 and section 5 deal with formulation of WLAPP and Experimentation. Conclusions are made in section 6 .

\section{FACE RECOGNITION IN A PARALLEL ENVIRONMENT}

The need of the hour is usage of parallel programming for every domain, so this is true for the research of image processing domain also. Multi-core and shared networking systems are increasing so their availability increases in the market. So every device is linked with parallel programming environment.

The problems such as more memory intensive, more computation and more data intensive can be solved by using Parallel programming. This Parallel programming provides more CPU resources, more memory resources, solve problems that were not possible with serial program and solve problems more quickly.

There are two basic parallel computer architectures can be used those are Distributed memory and Shared memory computer architectures. The issues of parallel programming includes reducing of execution time, computation time, idle time(waiting for data from other processors), communication time(time the processors take to send and receive messages), Load Balancing that divides the work equally among the available processors, Minimizing Communication to reduce the number of messages passed and amount of data passed in messages, where possible - overlap communication and computation and many problems scale well to only a limited number of processors.

Parallel Programming Models such as Directives-based parallel programming languages and Message passing (MPI) can be needed to discuss. In Directive-based parallel programming languages, most widely using language is OpenMP.

\subsection{MPI and open MP}

OpenMP is easier to program and debug than MPI, directives can be added incrementally (called gradual parallelization). It can still run the program as a serial code and serial code statements usually don't need modification. In this code is easier to understand and may be more easily maintained. But OpenMP can only be run in shared memory computers, requires a compiler that supports OpenMP and is mostly used for loop parallelization.

Message Passing Interface (MPI) passes messages to send/receive data between processes, and it is outgrowth of PVM software. MPI runs on either shared or distributed memory architectures that can be used on a wider range of problems than OpenMP. In this each process has its own local variables and distributed memory computers are less expensive than large shared memory computers. On the other hand MPI requires more programming changes to go from serial to parallel version, can be harder to debug and performance is limited by the communication network between the nodes.

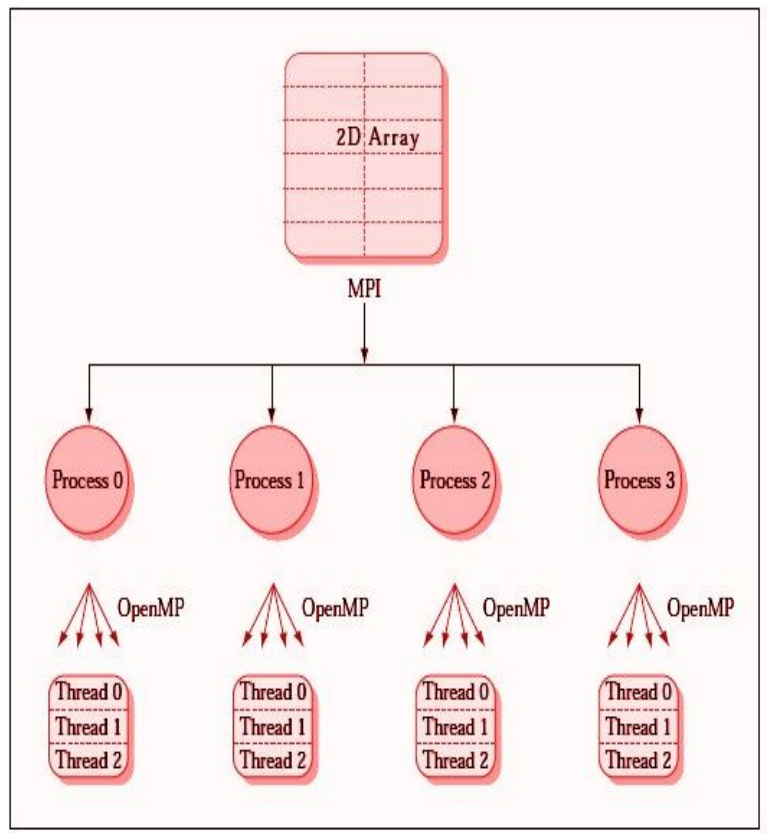

Figure 1: OpenMP and Message Passing Interface (MPI)

Figure 1 explains how MPI works in parallel and process' execution is shown with OpenMP.

\section{LOCAL ACTIVE PIXEL PATTERN (LAPP)}


Authors $[4,5,6]$ proposed LAPP and explained how to extract the Active pixels, which denote essential the of images and compared with other methods like LBP and performance evolution. Figure 2 gives neighbourhood.

The LAPP reduces the feature elements compared to LBP and also it reduces the computational time. Hence, the face recognition approach based on LAPP is quite suitable for both conventional and resource constrained environment. This LAPP addresses the issue limited resources such as memory and processing power. LAPP in parallel environment can be discussed in next section.

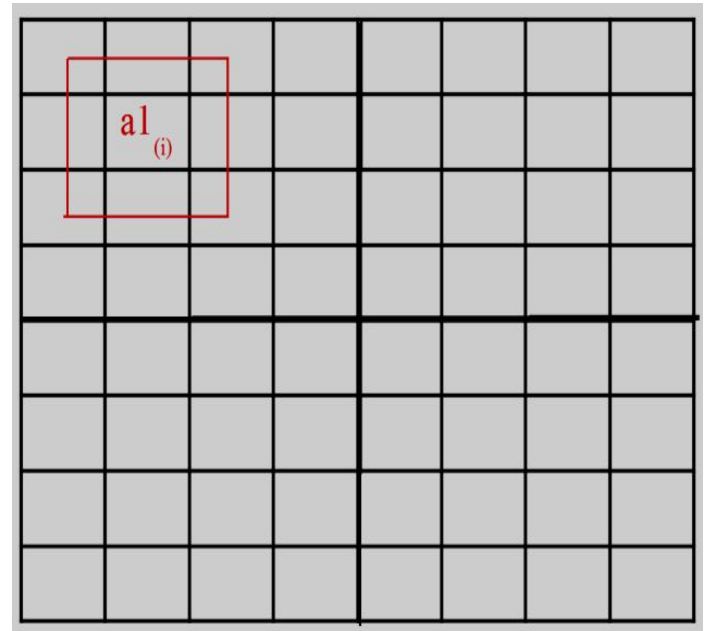

Figure 2: 8-Neighbourhood pixels of a Segment

\subsection{Parallel LAPP}

$>$ The parallel approach for LAPP is based upon its scalability.

$>$ The image division, neighbourhood extraction and computation are performed in parallel.

> For an image of size $256 \times 256$ is divided into $8 \times 8$ subregions using 1024 parallel workers.

> Each sub-region is then processed to compute active pixels using overlapping $3 \times 3$ neighbourhood mask.

> The parallel approach required 49 workers for each subject.

The figure 3 gives the implementation aspects.

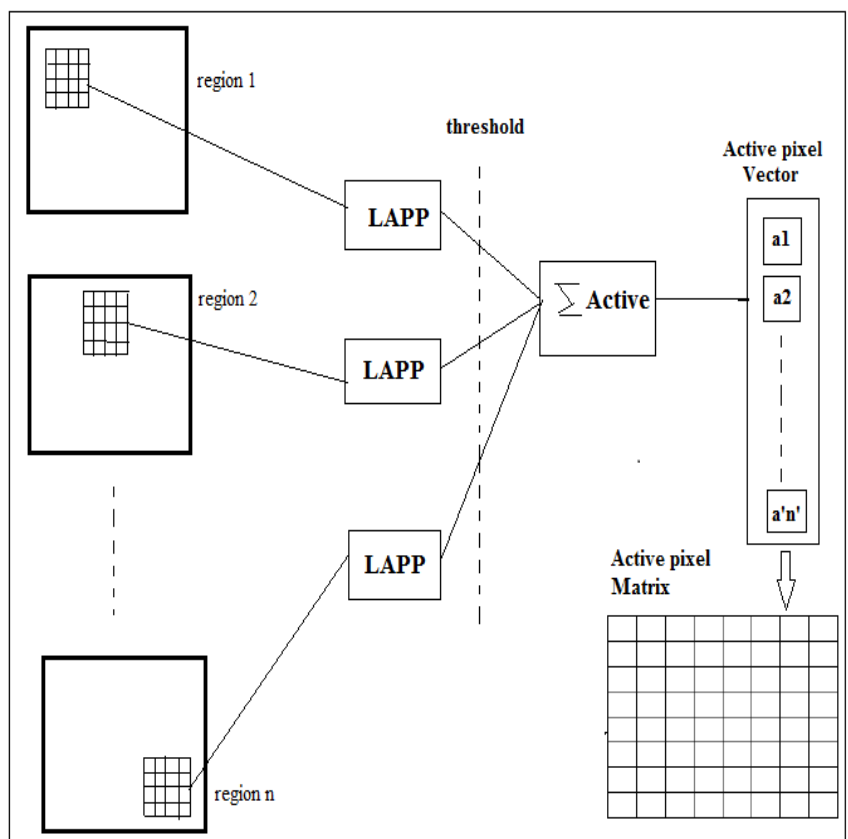

Figure 3: Local Active Pixel Pattern (LAPP) Feature

\section{Extraction}

\subsection{Active Pixel Computation using Parallel approach}

$>$ Broadcast $i^{\text {th }}$ sub-image to $i^{\text {th }}$ worker.

$>$ Computation of Active pixels by $i^{\text {th }}$ worker to $i^{\text {th }}$ subimage.

$>$ Gather the Active pixel count which will form the feature vector of the image.

\subsection{Parallel Template calculation}

$>\quad$ The subject template is calculating by adding corresponding elements of each image of concerned subject.

$>$ This is performed in parallel by 82 workers.

\section{WEIGHTED LOCAL ACTIVE PIXEL PATTERN} (WLAPP)

In order to get Weighted Local Active Pixel pattern, first computation of the weights for each region of the segmented image are needed and boost up these regions with corresponding weights. This process is explained below: 


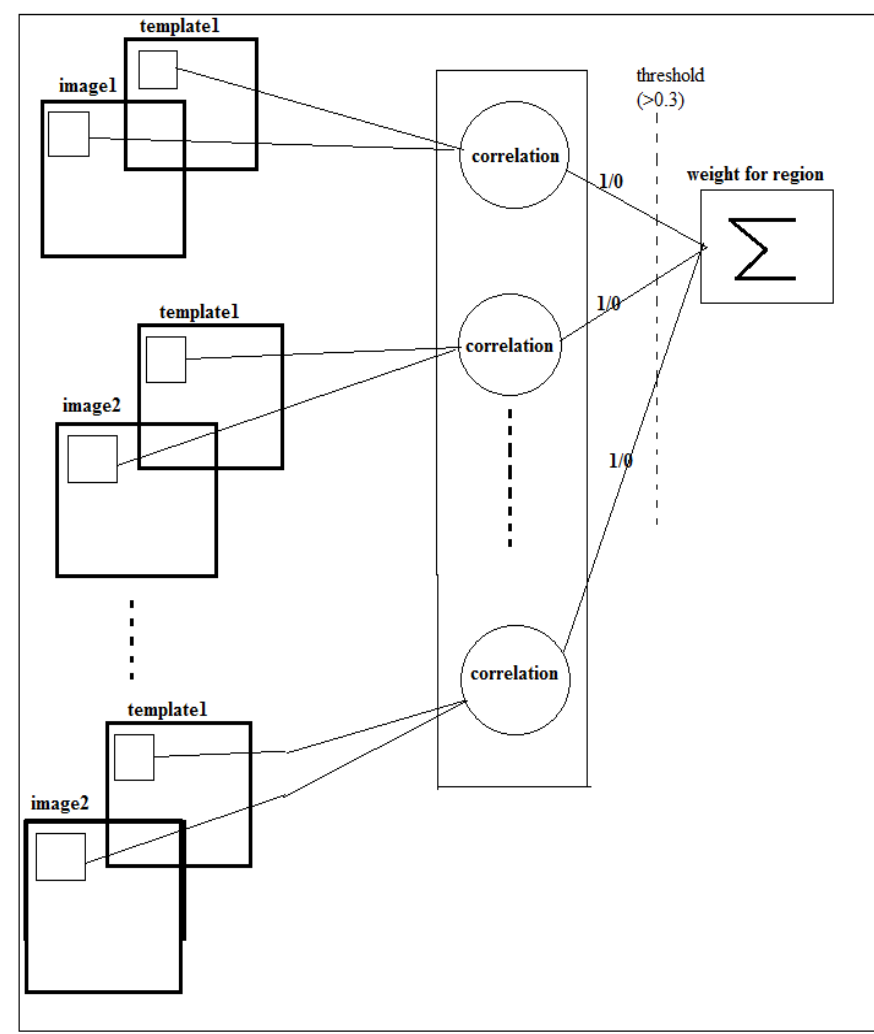

Figure 4: Weight Computation

\subsection{Weight computation}

$>$ Region-wise weight computation is done parallel for each subject by using broadcast correlation which contributes for the recognition (correlation strength is $>0.3$ ).

$>$ Here 343 workers are simultaneously performing for each subject means they work in parallel.

$>$ The figure gives the detailed process, how weight is computed for each region and how weight matrix is obtained.

Figure 4 reveal weight computation process while the

Figure 5 indicates the feature vector computation.

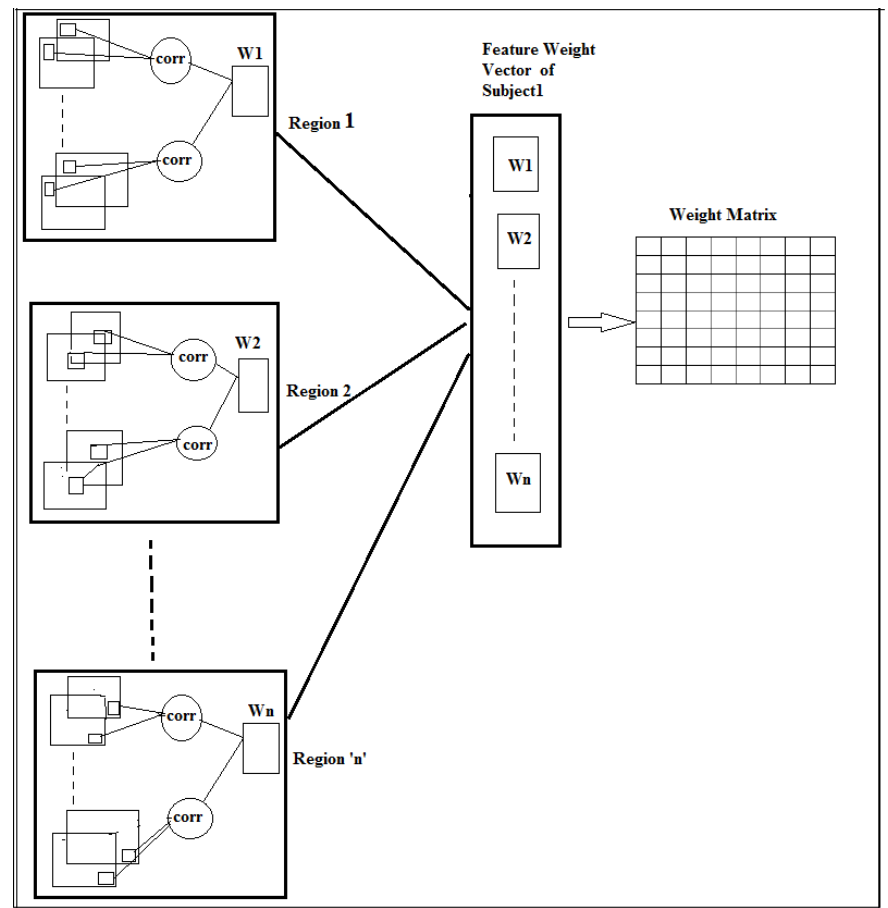

Figure 5: Feature Weight Vector for a subject

\subsection{Computation of WLAPP}

$>\quad$ The weight is given to each region of image gives Weighted Local Active Pixel Pattern (WLAPP).

$>$ For this Active Pixel Matrix elements are multiplied by corresponding Weights (element-wise multiplication, not the matrix multiplication). Note: If weight is zero, make it one because the original actual Active Pixels should not be disturbed.

$>$ Then Weighted Active Pixel Matrix and Weighted Template matrix, on which correlation of images can be performed (further discussed in Test of images), is obtained.

Figure 6 indicate the template weight computation.

\subsection{Testing of images:}

$>$ Broadcast test image to all workers.

$>$ Broadcast ith template to ith worker.

> Correlation by ith worker with ith template.

$>$ Gather the conclusions.

$>$ Index maximum correlation through worker is class.

Figure 7 reveal the parallel matching of test probe with templates. Feature vectors are used with this purpose. 


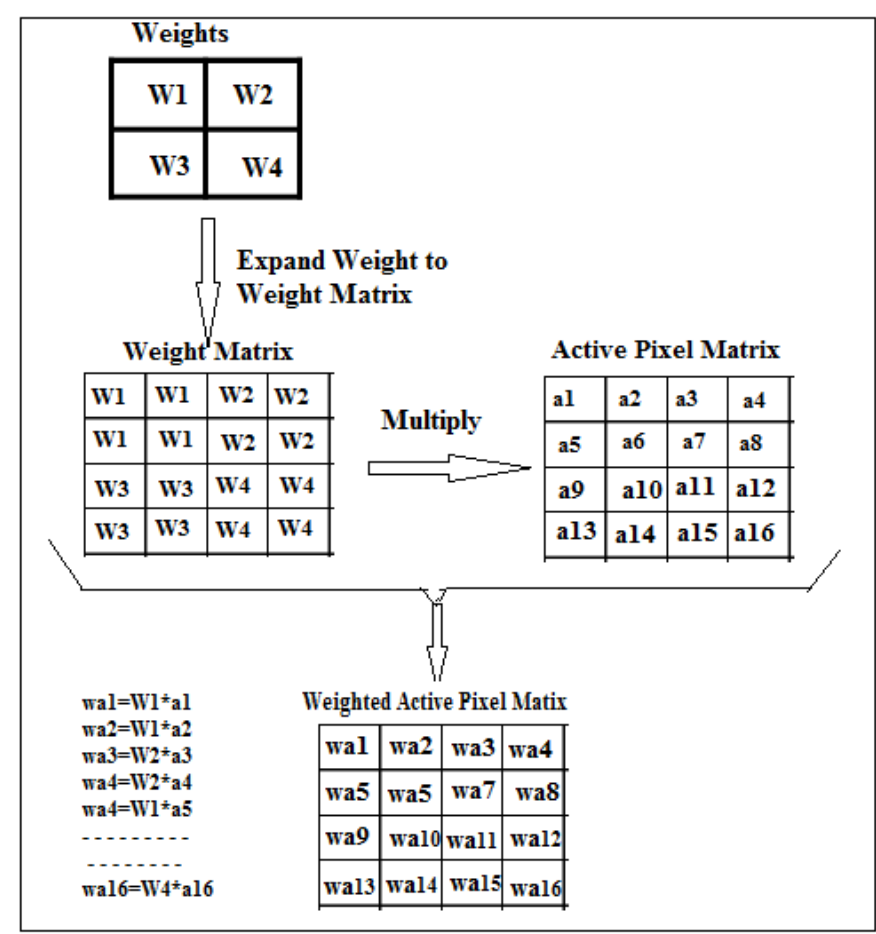

Figure 6: Weighted Local Active Pixel Pattern (WLAPP)

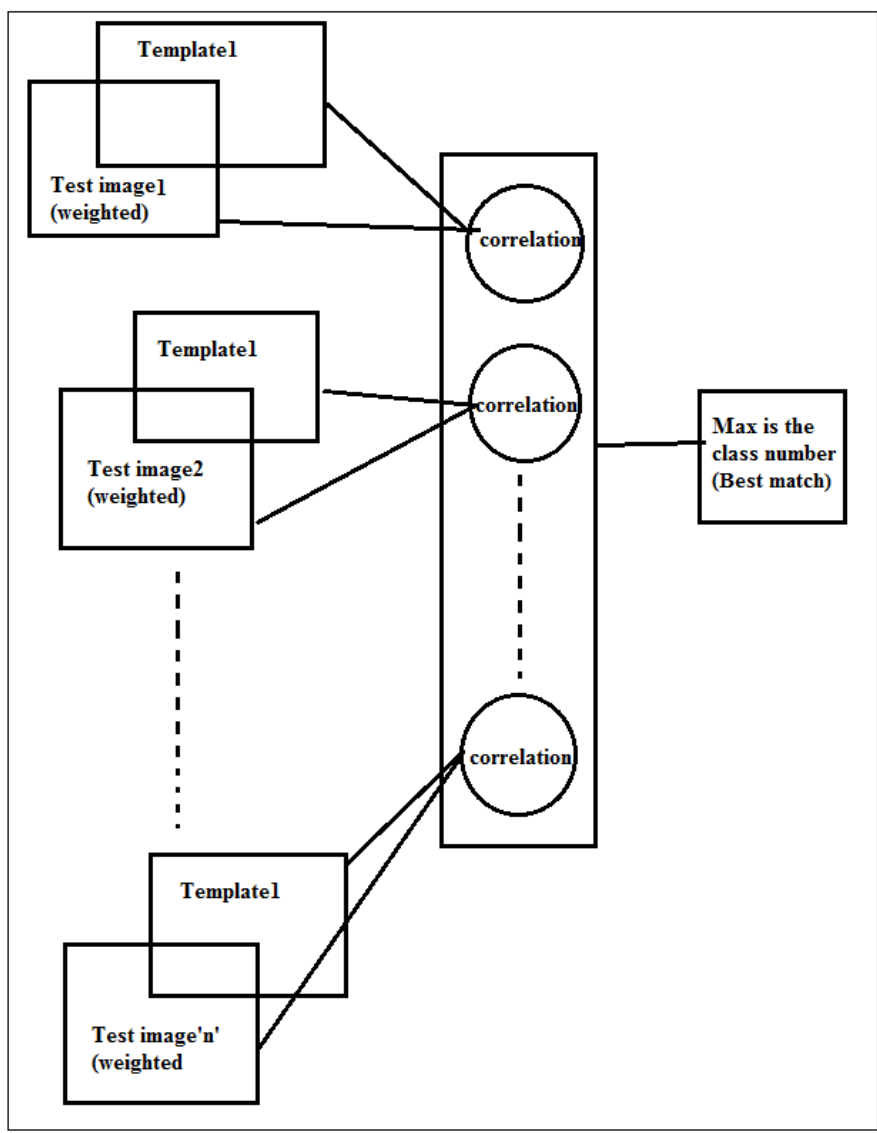

Figure 7: Text of Images (finding best match)

\section{Experimentation}

This experimentation is performed on the FG-Net Aging Database which has different types of images of different persons (called subjects) with varied poses, ages, expressions and illumination conditions.

\subsection{About FG-Net Aging Database}

It contains different sets of images belongs different persons of varying ages and Illumination conditions. For each subject (person), the dataset contains facial images are taken at different ages (0-65years) with different backgrounds and with different illumination conditions of pose variation and varying facial expressions. The total database contains totally 921 images of females and males of different age groups can be shown in below figure 8 .

\subsection{Experimentation Process}

The experimentation performed on this FG-Net Database poses good results. The experiment is performed on this database in following steps:

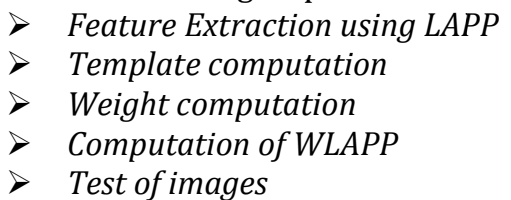

These all steps have discussed in section 4 .

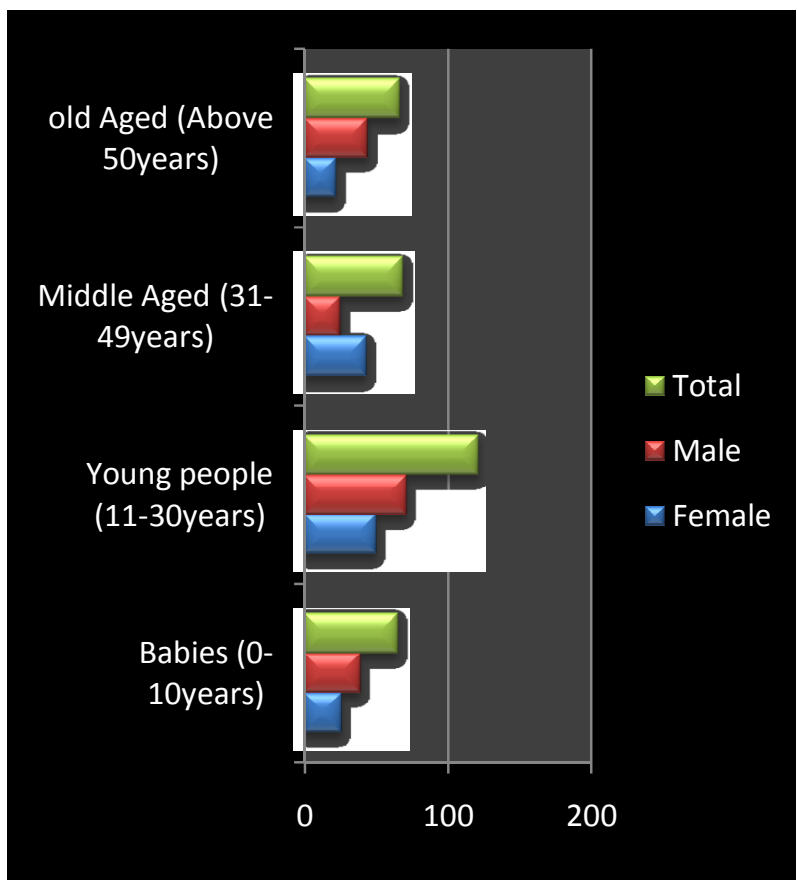

Figure 8: Dataset(FG-Net Database)

\subsection{Results and Discussion}

Weighted LAPP performance is compared with the other traditional methods in terms of recognition rate, training time and testing time. It was found that the WLAPP performance is highest compared to PCA, ICA and LAPP [4]. 
The overall convergence time is less for both LAPP and WLAPP (10.2sec) compared to ICA (1494sec) and PCA (15 sec). Table1 reveal this. In the Table 2 various size masks are used with different segmentations for various LAPP variants.

Table 1 : Performance comparison with other methods

\begin{tabular}{|c|c|c|c|c|}
\hline Performance & \multicolumn{4}{|c|}{ Face Recognition Methods } \\
\cline { 2 - 5 } Attribute & PCA & ICA & LAPP & WLAPP \\
\hline Accuracy & 91 & 93 & 93.8 & 95.7 \\
\hline Training Time & 9.4 & 1493 & .2 & .2 \\
\hline Testing Time & 6 & 1 & 10 & 10 \\
\hline
\end{tabular}

Table 2: Result Comparison

\begin{tabular}{|c|c|c|c|c|c|}
\hline \multirow[t]{2}{*}{ Size } & \multirow{2}{*}{$\begin{array}{l}\text { Segme } \\
\text { ntatio } \\
n\end{array}$} & \multirow{2}{*}{$\begin{array}{l}\text { Mas } \\
\mathrm{k}\end{array}$} & \multicolumn{3}{|c|}{ Correct Rate(LAPP) } \\
\hline & & & $\begin{array}{l}\text { Without } \\
\text { Boosting }\end{array}$ & $\begin{array}{l}\text { With } \\
\text { Boosting }\end{array}$ & $\begin{array}{l}\text { Weighted } \\
\text { Boostin } \\
\text { g }\end{array}$ \\
\hline \multirow{5}{*}{$\begin{array}{l}256 x \\
256\end{array}$} & $4 \times 4$ & $3 \times 3$ & 91.09 & 90.98 & 92.18 \\
\hline & \multirow[t]{2}{*}{$8 \times 8$} & $3 \times 3$ & 71.55 & 75.67 & 79.58 \\
\hline & & $5 \times 5$ & 80.99 & 78.39 & 95.98 \\
\hline & \multirow[t]{2}{*}{$16 \times 16$} & $3 \times 3$ & 55.26 & 62.10 & 65.03 \\
\hline & & $5 \times 5$ & 59.60 & 60.47 & 82.84 \\
\hline
\end{tabular}

These results are encouraged to look into more credibility so that the testing process is extended to some more images.

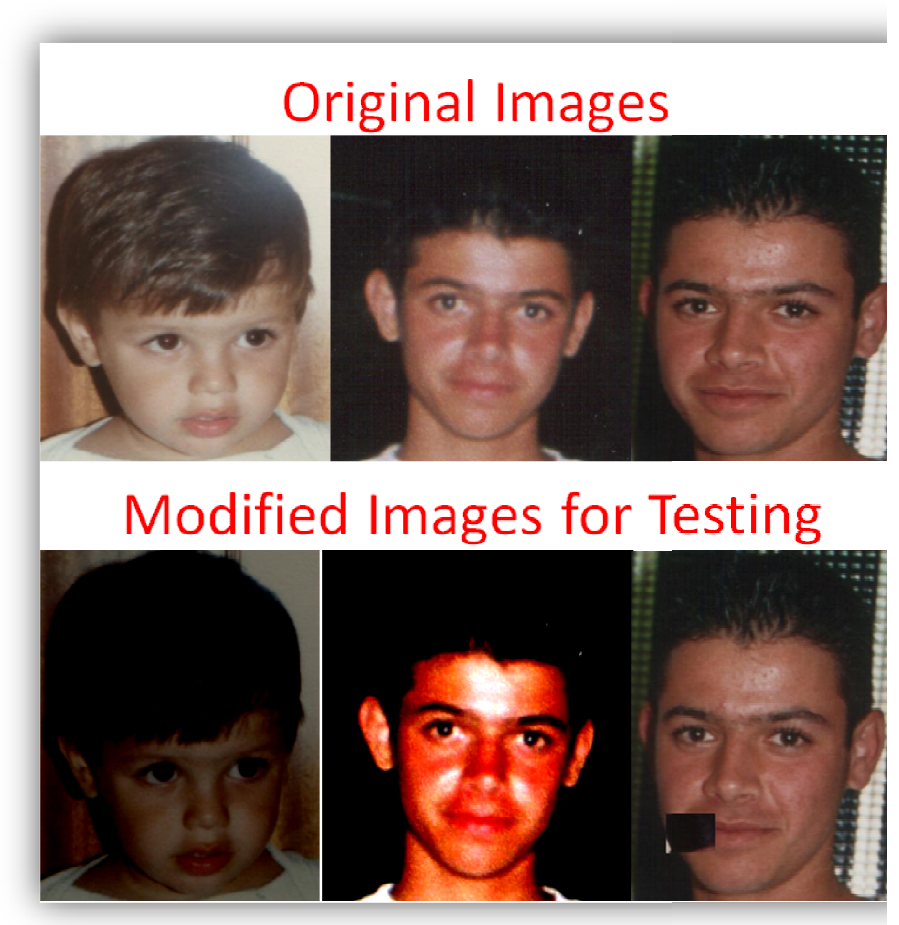

Figure 9: Testing with Modified images sample
By taking 3 images of each subject (person) and grouping them into 3 sets. For one set of images brightness is decreased, for second set contrast is increased and for third set an occlusion is added, this makes more than $20 \%$ distortion to original images. As Weighted Templates and Weights for those subjects are existed, Weighted Boosting is performed on them and Testing images and correlation is performed on those weighted Boosting images and Weighted Templates give us Best match (as discussed earlier). The results obtained are shown in Table 3.

Table 3: Artificially perturbed Test Probes \& Recognition Rate

\begin{tabular}{|l|l|l|l|l|l|}
\hline Size & $\begin{array}{l}\text { Segm } \\
\text { entat } \\
\text { ion }\end{array}$ & Mask & \multicolumn{3}{|c|}{ Correct Rate(LAPP) } \\
\cline { 4 - 6 } & $\begin{array}{l}\text { Without } \\
\text { Boosting }\end{array}$ & $\begin{array}{l}\text { With } \\
\text { Boosting }\end{array}$ & $\begin{array}{l}\text { Weighte } \\
\text { d } \\
\text { Boosting }\end{array}$ \\
\hline $\begin{array}{l}256 \times 25 \\
6\end{array}$ & $8 \times 8$ & $5 \times 5$ & 57.31 & 54.87 & $\mathbf{9 1 . 8 6}$ \\
\hline \\
6. CONCLUSION
\end{tabular}

The WLAPP is found to be attractive in terms of recognition rate. Performance comparison is given in Table 1 WLAPP gives better performance $(95 \%)$ is the best accuracy when comparing with the other approaches. Table 2 gives the correct recognition rate with artificial perturbed test probes. Concept can be extended to the mobile environment also (for details see [6]).

When this WLAPP is can be compared with other methods gives the best performance on the databases with various intensities, various illumination conditions, pose variations and different expressions unlike any other method as all other methods can overcome one of these constraints only. Comparison can be invited to discuss pros and cons of this approach as there is a wide area and lots of scope in this area and in image processing.

\section{REFERENCES}

[1] J. Cho, B. Benson, S. Mirzaei, R. Kastner, "Parallelized Architecture of Multiple Classifiers for Face Detection," Proc. IEEE International Conference on Application-specific Systems, Architectures, and Processors, 2009, pp. 75-82, ISSN :2160-0511.

[2] Kresimir Delac, Mislav Grgic and Marian Stewart Bartlett's Recent Advances in Face Recognition, ISBN 978-953-7619-34-3, Hard cover, 236 pages Publisher: InTech.

[3] Karm Veer Arya, Viraat Singh, Pabitra Mitra, Phalguni Gupta, Face Recognition using Parallel Associative Memory, 2008 IEEE International Conference on Systems, Man and Cybernetics (SMC 2008).

[4] Mallikarjuna Rao G et al., Face Recognition Applications using Active Pixels, International Journal of Engineering Research and Applications (IJERA) ISSN: 2248-9622 www.ijera.com Vol. 2, Issue 4, July-August 2012, pp.466-477.

[5] Mallikarjuna Rao G. et al.; Local Binary Patterns for Efficient Face Recognition, International Journal of Science and Engineering, Vol. 2(2):22-26, Dec. 2011, ISSN: 2086-5023.

[6] Mallikarjuna Rao G et al., An Investigation into the Use of Partial Face in the Mobile Environment, ISVC 2, volume 6939 of Lecture Notes in Computer Science, page 526-535, Springer, (2011). 